\title{
PROPUESTA DE INVESTIGACIÓN FORMATIVA EN LAS I.E.S Y SU APLICACIÓN EN LA ESCUELA COLOMBIANA DE CARRERAS INDUSTRIALES - E.C.C.I.
}

Profesor: Guillermo Roa*

\section{RESUMEN}

La Investigación formativa se considera la base para generar una cultura investigativa que conlleve a procesos de investigación propiamente dicha en instituciones de educación superior. Este proceso se encuentra fundamentado en el continuo mejoramiento y la calidad de la educación superior, en función de la capacitación, proponiendo generar propuestas nuevas e innovadoras, las cuales aporten en el desarrollo de la investigación y la creatividad de las nuevas generaciones de técnicos, tecnólogos y profesionales en Colombia. El punto de interrelación entre la docencia y la Investigación, tiene como resultado el aprendizaje por descubrimiento y construcción, centrada en el estudiante y con la renovación de la práctica pedagógica por parte del docente. El estudiante, busca, construye y organiza su aprendizaje, con base en los conocimientos existentes.

La investigación formativa es un proceso cambiante y dinámico demostrando que la educación en Colombia, ha evolucionado de manera directa, generando construcción educacional, desde las diferentes competencias de los estudiantes. La Facultad de Ciencias Económicas y Administrativas de la ECCl, pretende proponer un modelo de educación formativa, basado en competencias, este modelo se deberá estructurar en forma gradual.

La Escuela Colombiana de Carreras Industriales institución pionera en Colombia en la formación por ciclos propedéuticos, cuyos ciclos parten desde el nivel tecnología, debe permitir a los estudiantes ir adquiriendo las competencias investigativas necesarias a medida que avanza en su preparación universitaria según se establece en el Artículo No. 6 del Decreto 2566 de 2.003.

Dentro de las principales bondades y competencias que contribuye la Investigación Formativa, se puede mencionar el fomento a la argumentación, comunicación, la aplicación, la reflexión, el análisis y el ser propositivo.

*Administrados de Empresas, Docente de la Universidad ECCI. Correo. guilloroa@gmail.com 


\begin{abstract}
Formative education is considered as the bases needed to generate a research culture which leads to real research processes in higher education institutions (H.E.I.). This process has its foundations in the continuous improvement and quality of higher education, in function of training, suggesting to generate new and innovative proposals, which provide ideas in the development of research and the creativity of new generations of Colombian technicians and professionals.

The point of relation between teaching and research has as a result the learning by means of discovering and construction, centered in the student, with the renovation of the teacher's pedagogical practicum. The student searches, builds and organizes this with his previous knowledge.

Formative research is a changing and dynamic process showing that education in Colombia has evolved in a direct manner, generating an educational construction starting from the different students' competences. ECCl's faculty of economical and administrative sciences intends to propose a model of formative education, based on competences, this model should be structured in a gradual way.

The Escuela Colombiana De Carreras Industriales pioneer institution in the training of propaedeutic cycles, whose cycles star from the technologic level it must allow the students to acquire the research competences needed as he or she advances in his or her college as it is stablished in article 6 of decree 2566 of 2003.

Amongst the main goodness and competences with which the formative research contributes, can be mentioned the promotion of argumentation, communication, application, reflection, analysis and proactivity.
\end{abstract}

\title{
PALABRAS CLAVE
}

Investigación formativa, niveles de formación, ciclos propedéuticos, capacitación, I.E.S.

\section{INTRODUCCIÓN}

La globalización e internacionalización de los procesos económicos ha dinamizado la vida y la cotidianidad del mundo, la integración de la tecnología en el aula de clases, ha obligado a implementar una estructura educativa moderna. La educación como todos los aspectos ha cambiado y tomado rumbos de estrategia valorativa generada desde las potencialidades del individuo y su entorno.

La educación superior muestra un sendero diferente lleno de tecnología y mediática. Centrados en cambios severos que el individuo debe prepararse para generar interacciones crecientes y no quedarse estancado en la indiferencia y la vocación de conformidad.
El filósofo español Fernando Savater (2007) cree que La educación del ciudadano es el fundamento para que las sociedades democráticas se libren de vivir bajo el temor "a la influencia de los ignorantes". 


\section{CAPITULO I DEFINICIONES, NORMATIVIDAD}

\section{El proyecto de Investigación}

La palabra "Investigación" es el de averiguar una cosa, indagar o buscar. No obstante, en el medio académico este significado está enmarcado en el seguimiento de un método para designar un proceso sistemático, organizado y objetivo, cuyo propósito es responder a una pregunta o dar soluciones a problemas de nuestro entorno. Por lo tanto, es necesario considerar que aquellos que se dedican a la investigación desarrollan una estructura de pensamiento creativo, reflexivo y crítico.

Se puede decir que la base del proceso de investigación es el proyecto, el cual se considera una herramienta para precisar soluciones concretas, sobre el fundamento de una viabilidad económica, social, tecnológica y ambiental. Este es la célula básica de la planeación, permitiendo aunar las inversiones para alcanzar las metas de bienestar y desarrollo.

Para la presentación de un proyecto de investigación, no solo se debe mantener un orden, sino que los contenidos de cada segmento deben reflejar una serie de condiciones. En la introducción y justificación del proyecto se establecerán la importancia de la propuesta con respecto al campo del cual se ocupa y la relación con el desarrollo del tema en los últimos años; en el marco conceptual, se dejarán claros los fundamentos teóricos y los antecedentes del problema que ayudarán a comprender la base de pensamiento de por qué y cómo se utilizarán ideas y trabajos de otros investigadores; en los objetivos se definirán los propósitos y metas que respondan a las preguntas qué y para qué, coherentes con el planteamiento del problema y realizables según el marco conceptual y la metodología propuesta; en la metodología se presentará en forma organizada y precisa él cómo se alcanzará cada uno de los objetivos propuestos, la selección de fuentes de información, los instrumentos de recolección y procesamiento de información; en los resultados esperados se describirán los resultados teóricos o prácticos, los impactos y los posibles beneficiarios; en las estrategias de divulgación se hará explicito el compromiso de publicaciones o informes técnicos especiales y los medios para divulgar o transferir tecnologías y conocimientos a los presumibles beneficiarios y a la sociedad en general.

Finalmente, el cronograma de actividades se presentará una secuencia de actividades y el tiempo previsto para su realización, y el presupuesto se presentará una relación entre rublos, cantidades y montos solicitados según la metodología y horizonte del proyecto.

\section{Naturaleza de la Investigación Formativa}

La Investigación Formativa es una experiencia pedagógica que va más allá de la formulación de uno o varios ejercicios en la clase para que sean resueltos por los alumnos. Constituye un recurso educativo de valor que se vincula con profundidad al proceso de enseñanza-aprendizaje, enriquece el clima del aula y motiva extraordinariamente a los estudiantes en el camino de llegar al conocimiento y apropiarse de él. El entusiasmo que despierta en ellos libera en cierta forma al docente del peso de atraer la atención de su público y de responder completamente por el resultado. La responsabilidad de aprender pasa al alumno gracias a este recurso.

El maestro accederá gradualmente a programas cada vez complejos de Investigación Formativa, a medida que adquiera destreza en la dirección y el manejo del proceso. Sólo tiene que dar el primer paso para comenzar. Por ejemplo, en primera instancia, puede vincularla a su proceso de evaluación. De esta manera, los productos que resulten del trabajo con sus alumnos serán un elemento concreto que facilitará la calificación. Más adelante, cuando exista un mayor conocimiento del recurso, podrá vincularlo a su clase como una herramienta educativa. Ayudará 
al abordaje de las nociones más complejas de la materia que dicta al generar en torno a ellas procesos de indagación que conducen a una apropiación más vivencial del conocimiento.

Finalmente, cuando se tenga mayor experiencia en el uso de este medio, podría darse el paso de vincularlo a su actividad docente como un método pedagógico que impulsa el conjunto de temas que integran los espacios académicos.

\section{Requisitos}

La Investigación Formativa puede confundirse con las tareas o trabajos que tradicionalmente se proponen a los estudiantes en la clase. Sin embargo, se considera que esta debe ir más allá. Mientras una tarea cumple con el propósito de lograr que el alumno practique nociones aprendidas y habilidades adquiridas, la Investigación Formativa motiva procesos de indagación donde los alumnos utilizan el método científico para llegar hasta lo que constituye para ellos nuevo conocimiento.

Por esta razón, para que un trabajo en clase alcance el nivel de Investigación Formativa y se considere válido pedagógicamente debe cumplir con tres requisitos esenciales:

1. Será formulado como un proyecto de investigación que se desarrollará a lo largo de uno o varios semestres.

2. El proceso seguido por los alumnos para llegar al conocimiento será establecido en el proyecto y debe quedar documentado a medida que avance. Es decir, se requiere dejar constancia de cada una de las etapas principales a través de un informe que indique cómo se hizo y qué se obtuvo.

3. El trabajo generará uno o más productos finales, susceptibles de ser difundidos (socializados) en la comunidad académica o en otros escenarios. En lo posible, se busca que esos productos tengan alguna relación de beneficio con la comunidad donde está ubicada la universidad o con otras comunidades.

\section{Lineamientos de la Investigación Forma- tiva en la Escuela de Carreras Industriales y la Facultad de Ciencias Económicas y Administrativas}

Conferencia Mundial sobre la Educación, UNESCO (1998) consideró que²:

Parte esencial de la naturaleza de la Educación Superior en nuestro tiempo es la obtención de nuevos conocimientos, ya sea en términos locales o globales. Esto hace que la investigación adquiera para la academia una función capital, que cada día es más evidente y necesaria. En las conclusiones de la Conferencia Mundial sobre Educación Superior de UNESCO, de octubre de 1.998, realizada con el propósito de concretar acciones prioritarias para el nuevo siglo en este campo, se insistió en que una de las grandes misiones de la Educación Superior es obtener y difundir nuevo conocimiento.

En el aparte dedicado a "Misiones y Funciones de la Educación Superior", parágrafo " $\mathrm{g}$ " del artículo $1^{\mathrm{a}}$, la Conferencia concluye que es necesario en la Educación Superior de todos los países "promover", generar y difundir conocimientos por medio de la investigación y, como parte de los servicios que ha de presentar a la comunidad, proporcionar las competencias técnicas adecuadas para contribuir al desarrollo cultural, social y económico de las sociedades, fomentando y desarrollando la investigación científica y tecnológica a la par que la investigación en el campo de las ciencias sociales, las humanidades y las artes creativas.

EI CNA (1998) afirma3 :

La investigación universitaria es un proceso de búsqueda de nuevo conocimiento, proceso caracterizado por

${ }^{2}$ Conferencia Mundial sobre Educación, UNESCO 1998 
la creatividad del acto, por la innovación de ideas, por los métodos rigurosos utilizados, por validación y juicio crítico de pares.

A la investigación está unida íntimamente la creatividad ya que en buena medida los resultados de la investigación son también creación de conocimiento o de tecnología. Y la investigación científica debe operar en la universidad no sólo en el ámbito de las disciplinas o ciencias básicas, sean éstas naturales, formales o sociales, sino también en el ámbito de las profesiones o carreras. Ahora, en la ECCl, en el Acuerdo No. 02 del 28 de Marzo de $2.008^{4}$, en Capítulo I Visión, Definiciones en su Artículo 1 la concepción de la Investigación, ciencia y tecnología con producción original, experimentación, pensamiento creativo y el modelo pedagógico, expresados en el Proyecto Educativo Institucional; y los propósitos y referentes del Sistema Nacional de Ciencia y Tecnología, con el fin de garantizar el cumplimiento de la política, lineamientos y objetivos establecidos para el proceso de investigación de la ECCI.

En el Artículo 2. Definiciones: Para los efectos del presente reglamento de investigación, se entiende por:

- Ciencia: Conjunto de conocimientos obtenidos mediante la observación y el razonamiento, sistemáticamente estructurados y de los que se deducen principios y leyes generales.

- Tecnología: Conjunto de teorías y de técnicas que permiten el aprovechamiento práctico del conocimiento científico, para diseñar, generar o manufacturar un bien o un servicio.

- Técnica: Conjunto de saberes prácticos procedimientos para obtener un resultado. Requiere de destreza manual e intelectual, y generalmente con el uso de herramientas. Las técnicas se transmiten de generación en generación.
- Innovación: Es un proceso de gestión de cambios específicos en procesos, productos, ideas, materiales, prácticas educativas y/o empresariales, que buscan el perfeccionamiento y mejoramiento, con miras al desarrollo personal, organizacional y social.

- Investigación: " la investigación es un procedimiento reflexivo, sistemático, controlado y crítico que tiene por finalidad descubrir o interpretar los hechos y fenómenos, relaciones y leyes de un determinado ámbito de la realidad. -una búsqueda de hechos, un camino para conocer la realidad, un procedimiento para conocer verdades parciales,-o mejor-, para descubrir no falsedades parciales."(Ander-Egg, 1992).

- Investigación Científica: Conjunto de búsquedas, estudios y experimentación, enfocados a la generación de conocimiento con base en la aplicación rigurosa del método científico.

- Investigación Tecnológica: Conjunto de estudios, observaciones y experimentación, enfocados a la generación de una nueva tecnología (innovación tecnológica), con base en conocimientos científicos y/o la experiencia acumulada en la práctica productiva, las buenas prácticas de manufactura y el conocimiento acumulado en el servicio técnico a clientes.

También incluye la investigación realizada sobre tecnologías ya existentes para su mejora u optimización y la investigación en áreas de la administración de la tecnología.

- Investigación Social: La investigación social es el proceso que, permite obtener nuevos conocimientos en el campo de la realidad social, o bien estudiar una situación para diagnosticar necesidades y problemas a efectos de aplicar los conocimientos con fines prácticos (investigación aplicada). 
- Desarrollo tecnológico: Se entiende por desarrollo tecnológico, el proceso de generación de una tecnología con el fin de establecer un nuevo sistema productivo para la manufactura de un bien o servicio, así como el cambio de productos o procedimientos en sistemas productivos ya establecidos.

\section{Uso de TIC en la Investigación Formativa}

Según Conferencia Mundial de Educación Superior de (2009): "La aplicación de las TIC a la enseñanza y el aprendizaje encierra un gran potencial de aumento del acceso, la calidad y los buenos resultados. Para lograr que la aplicación de las TIC aporte un valor añadido, los establecimientos y los gobiernos deberían colaborar a fin de combinar sus experiencias, elaborar políticas y fortalecer infraestructuras, en particular en materia de ancho de banda" Igualmente, asegura que "Habida cuenta de la investigación y la innovación mediante iniciativas conjuntas de múltiples copar cipes entre el sector público y el privado, que abarque a las pequeñas y medianas empresas". (UNESCO, 2009).

Con la llegada de las nuevas tecnologías de la información (TIC por sus siglas en inglés) se llevan a cabo una serie de cambios en la sociedad. Los impactos que se han originado por el uso de las TIC en la educación, se pueden mencionar en tres grupos: (Majó 2000)

\section{a. La enseñanza del uso de las propias tecnologías}

b. El uso de las tecnologías como medio de apoyo para los contenidos existentes.

c. La adaptación del sistema educativo para la nueva realidad social que se dará a raíz del uso de las tecnologías.

Estas tecnologías hacen parte de la sociedad y se incorporan a ella en la cotidianeidad. Para la Escuela Colombiana de Carreras Industriales ECCI-ET no es desconocida la utilización de estas tecnologías y la apropiación de estas en función de la formación de nuestra comunidad académica, como por ejemplo la herramienta Aulas Virtuales.

\section{Propuesta}

La Investigación Formativa puede estar antecedida por una propuesta que se entregue desde la Facultad de Ciencias Económicas y Administrativas En la propuesta él debe plantear lo que desea hacer en uno o más semestres en este campo, sustentando la importancia del programa en sus aspectos pedagógico e investigativo y demostrando la viabilidad que existe para hacerlo. Los lineamientos seguidos en las Políticas Públicas sobre Educación Superior: ciclos y competencias del 21 de Agosto 2.007, en las cuales se hace referencia a la investigación formativa como la forma para que se desarrolle una cultura investigativa que conlleve a un pensamiento crítico y autónomo que permita a estudiantes y profesores acceder a los nuevos desarrollos del conocimiento y la tecnología. Así mismo se afirma que "un programa de cualquier nivel ofrecido por una universidad deberá tener a la investigación como sustento".

Para concretar las acciones en el Artículo 6 Decreto 2566 - 2003 están claramente definidas las competencias requeridas por niveles donde se espera que:

6.1 El tecnólogo deberá ser capaz de desarrollar en forma autónoma investigación descriptiva y correlacional, que involucren al menos un número bajo de variables.

6.2 El profesional universitario sea capaz de desarrollar en forma autónoma investigación correlacional y explicativa en las áreas de su disciplina o profesión.

\section{Ejes temáticos y núcleos problémicos}

En la Investigación Formativa se entiende que la apropiación del nuevo conocimiento por parte del alumno se asemeja a un proceso de investigación.

De ahí que sea necesario que el profesor determine su propuesta/proyecto luego de examinar los ejes temáticos de su materia, determinando en ellos los núcleos problémicos. Es decir, aquellos conceptos que, por su complejidad y novedad, sean susceptibles de transformarse en una o varias preguntas de investigación. 


\section{Interdisciplinariedad}

Puede suceder cuando un profesor presenta un proyecto de Investigación Formativa y tiene la posibilidad de unirse con otros de su facultad (Comercio Exterior con Mercadeo y Publicidad, Gestión Contables en la parte técnica, etc) de otras facultades (Ingenierías, Artes) para ampliar la cobertura y convertirlo en una propuesta/programa interdisciplinario.

Esto enriquece la investigación inicial con otros objetivos pedagógicos que refuerzan la motivación de los alumnos hacia el aprendizaje.

\section{Grupos de trabajo}

En la Investigación Formativa es conveniente integrar grupos de trabajo con los estudiantes, puesto que esto proporciona múltiples beneficios. En primer término, se preparan para los procesos modernos de investigación que tienden a no ser, en general, actos individuales y aislados. La obtención del conocimiento en un sistema científico con alto grado de desarrollo requiere del intercambio de saberes, de un flujo interdisciplinario que enriquezca el estudio y permita trabajar con mayor complejidad. En segundo, la formación de esos grupos asegura el crecimiento de cada uno de sus integrantes, puesto que se intercambian habilidades y destrezas, se despierta un mayor compromiso individual y se consigue un diálogo constructivo en torno a las estrategias y los pasos que se siguen para llegar al nuevo saber, desarrollando así competencias profesionales.

En tercer lugar, generar en el(os) alumno(s) las habilidades y destrezas indispensables que requiere para su posterior trabajo profesional, debido a que estará obligado a participar en grupos de trabajo en las empresas e instituciones a las que se vincule o en las comunidades con las que se relacione.

\section{Diferencia entre Tarea y Problema}

El profesor deberá diferenciar con claridad entre una tarea y un problema. La tarea o ejercicio no implica la apropiación por parte del alumno de nuevos conocimientos. Se trata simplemente de la puesta en práctica de nociones ya adquiridas y la ejercitación de habilidades y destrezas que se tienen.

En cambio, la formulación del problema conduce al alumno al conocimiento nuevo y lo lleva a desarrollar otras habilidades y destrezas para llegar a él, que hacen parte del método científico.

La diferencia entre esas dos categorías depende del nivel de conocimientos que tienen los alumnos de la ciencia o disciplina donde está inscrita la clase. Lo que constituye un problema para los estudiantes de un semestre no sería más que una tarea para otros, que se encuentran en cursos más adelantados.

\section{Resultados esperados}

Los resultados esperados al finalizar cada semestre a tecnológico y profesional, deberán estar de acuerdo con los objetivos planteados por el un Grupo Gestor Asesor y los Lineamientos Institucionales, pero no son una reformulación de los mismos. Estos resultados serán de cuatro categorías:

1. Los que se esperan pedagógicamente en relación con la asignatura que orienta el profesor y con la formación misma del estudiante. Un programa de Investigación Formativa incluye, el desarrollo de habilidades y destrezas en el alumno que concluyen en competencias para resolver problemas, para encontrar nuevos conocimientos, para trabajar en grupo, para redactar documentos, para socializar los resultados obtenidos con su trabajo, etc.

2. Los referentes a los conocimientos que serán adquiridos y a su elaboración teórica posterior. Estos resultados se refieren a los ejes temáticos de la materia y a los núcleos problémicos que el docente ha propuesto con su proyecto que manejen los estudiantes. 
3. Los productos que se obtienen al alcanzar los objetivos específicos propuestos sea en forma de nuevo conocimiento, información, bienes o servicios. Estos productos, deberán ser concretos y verificables.

4. Los que se esperan frente a la comunidad académica y a la sociedad misma. Se ha indicado que uno de los beneficios de la Investigación Formativa consiste en llevar el conocimiento a la acción, buscando para la comunidad una mejor calidad de vida.

Es necesario indicar en los proyectos de investigación formativa cómo podrán identificarse y verificarse cada uno de los logros esperados.

\section{Estrategia de Difusión}

En un proyecto de Investigación Formativa es recomendable incluir una estrategia de difusión de los resultados, sobre todo en la comunidad académica de la Facultad en primera instancia. En especial, aquellos proyectos que tienen notoriedad por los aportes que brindan como proceso de investigación, realización pedagógica o integración interdisciplinaria.

La difusión cumple con varios objetivos. Muestra en la Escuela y/o Facultad un ejemplo de trabajo que se ha destacado y sea motivo de emulación en la facultad a la que pertenece el docente.

Estimula a los alumnos, ellos tienen la oportunidad de compartir con otros compañeros los productos obtenidos en la investigación. Es un logro importante del profesor que lo puede socializar a otros colegas como aporte a la reflexión que debe existir en el medio académico.

La socialización de los resultados de investigaciones formativas notables contribuye, además, a establecer estándares de calidad para esta clase de estudios dentro de la Escuela Colombiana de Carreras Industriales ECCI.

\section{Grupo Responsable de la Investigación}

En el proyecto deberá consignarse el nombre del profesor que promueve y dirige el estudio y los nombres de los alumnos que participan en él, indicando a qué grupos pertenecen y qué labor se asignará a dicho grupo.

\section{Cronograma de Actividades}

Incluye las actividades que se han programado y las fechas de la realización de cada una de ellas. La claridad en este aspecto es un medio para que los alumnos respondan a los tiempos establecidos y se entrenen en el cumplimento de un plan de trabajo.

\section{CAPITULO II. INFORMACIÓN, ACTIVIDADES}

La investigación formativa: involucra la promoción de la investigación en las instancias académicas, especialmente en docentes y estudiantes, de la ECCly la Facultad de Ciencias Económicas y Administrativas, desde dos vías:

1. Actitud investigativa, entendida como el desarrollo de competencias en el individuo que lo comprometan subjetivamente frente a los procesos de investigación: la curiosidad, la indagación, la visión crítica, la capacidad argumentativa e interpretativa, entre otros.

2. Apropiación del proceso de investigación como estrategia pedagógica; ello implica para:

- El estudiante, el aprendizaje de los procesos de investigación relacionados con las áreas de formación involucradas en su currículo académico.

- El docente, la transformación del quehacer pedagógico, haciendo uso real y efectivo del proceso de in-vestigación en la planeación, desarrollo y evaluación del acto pedagógico.

- El docente, la sistematización del proceso educativo entendiendo su práctica pedagógica como un problema de investigación que supone la actualización, la exploración sistemática y retroalimentación del currículo. 


\section{Actividades de Investigación Formativa (tipos de proyecto)}

15.1 Proyectos de Aula: Competencias en procesar y analizar información:

Desarrollo de contenidos de la asignatura. Son realizados por los estudiantes y coordinados por el docente. Ej: Asignatura Guión para documental, Tema: ¿Cuáles son los requisitos y procesos para estructurar un guión para documental?

\subsection{Proyectos Paralelos (autoestudio):}

Competencias en Planteamiento de inquietudes y desarrollo de conductas de auto estudio.

Desarrollo de temas complementarios a la asignatura o a la profesión. Son realizados por los estudiantes y evaluados por el docente. Ejemplo: Asignatura: Introducción a la Ingeniería de Sistemas. Herramienta pedagógica: Software KAREL.

\subsection{Proyectos de Aplicación en Asignaturas:}

Competencias en el planteamiento de un problema y en la definición de metodologías y en las técnicas de investigación más apropiadas. Se desarrollan a través de convenios con organizaciones solucionando un problema o buscando oportunidades sobre temáticas inherentes a la profesión. Son realizados por los estudiantes y direccionados por el docente. Ej. Asignatura Prácticas Empresariales. Estudios de públicos convenio con el Museo Nacional.

15.4 Semilleros de investigación: Competencias en la aplicación de conocimientos en metodologías adquiridas durante la carrera para responder a una necesidad especifica de una organización o comunidad.

Proyectos de investigación aplicada (Ej. Líneas de investigación, trabajos de grado). Grupos de investigación conformados por docentes y estudiantes. Ej. Opciones de grado: Planes de negocio para la creación de empresa.
15.5 Células Tecnológicas: Son convenios académicos con empresas (Ej. IBM, Microsoft), con el propósito que los docentes y estudiantes se apropien de la tecnología. Grupos de autoestudio conformados por docentes tutores y estudiantes. Ej. En ingeniería de sistemas algunas de las células se especializan en DB2, ORACLE, JAVA, CISCO.

\section{Las Competencias o acerca del Desarro- llo de la Alta Inteligencia a través del Método de las Ciencias}

Como bien dice Gadamer (1997), el método es más una puesta en práctica del saber, es decir, posibilita la formación; no es tanto llegar a tener un conocimiento de las cosas mismas sino llegar a tener una comprensión de la manera como hemos llegado a ser lo que somos, en una individualidad singular y concreción plena.

La puesta en práctica de un saber, que posibilita la formación, está ligada con el concepto de competencias, como el conjunto de condiciones necesarias para comprender las reglas y las estructuras de un conocimiento específico y poder interpretarlo, aplicarlo y transformarlo.

Epistemológicamente, una de las tantas clasificaciones de las ciencias, las estipula como formales, naturales y humanas. Cada acápite de ellas genera un método o forma de construir su saber que le ha sido propio por un lapso de tiempo, pero que hoy se circunscriben en una filosofía que se complejiza cada vez más.

Cada método genera unas posibilidades, en tanto competencias, para saber hacer en el contexto de las sociedades del conocimiento. Dichas competencias generan una gama de habilidades en tanto el saber relacionarse con a través de ciertas destrezas que algún sistema de tareas constituidas en estrategias didácticas, reconocidas en las comunidades académicas facilitan su adquisición en las nuevas generaciones. Veamos: 


\begin{tabular}{|c|c|c|c|c|c|}
\hline CIENCIAS & MÉTODO & COMPETENCIA & HABILIDADES & DESTREZAS & ESTRATÉGIAS \\
\hline Formales & Deductivo & Abstraer & Inferir & $\begin{array}{l}\text { Conjeturar } \\
\text { Conjeturar } \\
\text { Simbolizar } \\
\text { Formalizar }\end{array}$ & $\begin{array}{l}\text { Mapas conceptuales } \\
\text { Lúdica }\end{array}$ \\
\hline \multirow[t]{5}{*}{ Naturales } & Inductivo & Demostrar & $\begin{array}{l}\text { Relacionar } \\
\text { Comprender } \\
\text { axiomas } \\
\text { Argumentar } \\
\text { teoremas } \\
\text { Refutar }\end{array}$ & $\begin{array}{l}\text { Simbolizar } \\
\text { Formalizar } \\
\text { Describir } \\
\text { Relacionar }\end{array}$ & $\begin{array}{l}\text { Talleres } \\
\text { Mapas conceptuales }\end{array}$ \\
\hline & & Explicar & $\begin{array}{l}\text { Observar } \\
\text { Comparar } \\
\text { Clasificar }\end{array}$ & $\begin{array}{l}\text { Ver } \\
\text { Escuchar } \\
\text { Relacionar }\end{array}$ & $\begin{array}{l}\text { ABP } \\
\text { Salidad de campo } \\
\text { Trabajo de laboratorio }\end{array}$ \\
\hline & & Generalizar & $\begin{array}{l}\text { Crear leyes } \\
\text { Abstracción } \\
\text { Convalidad }\end{array}$ & $\begin{array}{l}\text { Constrastar } \\
\text { Refutar } \\
\text { Conjeturar }\end{array}$ & $\begin{array}{l}\text { Experimentación } \\
\text { Salidas de campo } \\
\text { Trabajo de laboratorio }\end{array}$ \\
\hline & & Pronosticar & $\begin{array}{l}\text { Hipotizar } \\
\text { Modelar } \\
\text { Inferir análizis de tendencias }\end{array}$ & $\begin{array}{l}\text { Manejo de datos } \\
\text { Calcular } \\
\text { Operar }\end{array}$ & $\begin{array}{l}\text { Heuristica* } \\
\text { ABP }\end{array}$ \\
\hline & & Modelar & $\begin{array}{l}\text { Abstraer } \\
\text { Simular } \\
\text { Diseñar }\end{array}$ & $\begin{array}{l}\text { Calcular } \\
\text { Operar }\end{array}$ & $\begin{array}{l}\text { Heuristica* } \\
\text { ABP }\end{array}$ \\
\hline \multirow[t]{4}{*}{$\begin{array}{l}\text { Sociales, Huma- } \\
\text { nas y del Espíritu }\end{array}$} & Herméneutica & Interpretar & $\begin{array}{l}\text { Comprender } \\
\text { Analizar } \\
\text { Sintetizar }\end{array}$ & $\begin{array}{l}\text { Leer } \\
\text { Escribir }\end{array}$ & $\begin{array}{l}\text { Seminario } \\
\text { Estudio de casos }\end{array}$ \\
\hline & & Comunicación & $\begin{array}{l}\text { Leer } \\
\text { Escribir } \\
\text { Escuchar } \\
\text { Hablar }\end{array}$ & $\begin{array}{l}\text { Aplicar reglas de } \\
\text { la gramática }\end{array}$ & Seminario \\
\hline & & Escritura & Leer & $\begin{array}{l}\text { Aplicar reglas } \\
\text { ortográficas }\end{array}$ & Ensayo \\
\hline & Dialéctica & $\begin{array}{l}\text { Tesis Antisis } \\
\text { Antisis } \\
\text { Sintesis }\end{array}$ & $\begin{array}{l}\text { Retótica } \\
\text { Argumentación }\end{array}$ & $\begin{array}{l}\text { Manejo de la voz } \\
\text { Escucha }\end{array}$ & Conversatorio \\
\hline
\end{tabular}

Tabla No. 1 RELACIÓN CIENCIAS, MÉTODOS COMPETENCIAS Y ESTRATEGÍA DIDÁCTICA.

- Para alguno teóricos la heurística es un método para otros es una estrategia. Según la perspectiva y la claridad con la cual se utilice puede ser ambas.

\section{CONCLUSIONES DE LA INVESTIGACIÓN EN EL AULA}

- No todos los trabajos o documentos que hacen los estudiantes son de investigación.

- Es necesario un uso riguroso del lenguaje, que además de ser parte de una cultura académica seria, ayuda a precisar qué actividades son pertinentes para el desarrollo del pensamiento científico.

- La mejor manera de avanzar es partir de la experiencia adquirida y someterla a discusión y evaluación.

- Formación de docentes, seguimiento y asesoría.

\section{A MANERA DE CONCLUSIONES GENERALES}

La estructura pedagógica basada en investigación, con el ánimo de incrementar las competencias es un eje central en la formación de tecnólogos y profesionales en la Escuela de Carreras Industriales - ECCI, brindando el soporte para desarrollar un aprendizaje de permanente cuestionamiento y se convierte en un ejercicio autónomo y cotidiano en nuestros estudiantes y docentes.

Este proceso los lleva a un cambio de actitud y valores teniendo como base el conjunto de conocimientos, habilidades, disposiciones y conductas que le permiten la realización exitosa de sus actividades.

La Facultad de Ciencias Económicas y Administrativas ha estado motivado permanentemente en la construcción de un proceso en el cual lo pedagógi- 
co y lo tecnológico se unan en beneficio tanto de estudiantes como de docentes y donde el centro de formación sea el estudiante, mediante la realización de documentos de investigación formativa.

Por tal motivo el principal derrotero es llevar a cabo un seguimiento con análisis y diagnóstico de la calidad de los resultados de la investigación realizada por los estudiantes en aras de construir un modelo de autonomía intelectual que sea útil a los futuros tecnólogos y profesionales y que ofrezca un soporte al énfasis pedagógico de la Facultad de Ciencias Económicas y Administrativas.

El modelo pretende generar una disposición al trabajo en equipo, dando origen a grupos, integrados por docentes investigadores de diferentes disciplinas, que muestran como resultado procesos de aprendizajes alrededor de documentos comunes. Así como también ha avanzado:

- Las funciones de docencia e investigación en la educación superior necesitan interrelacionarse desde la perspectiva de los procesos investigativos.

- La investigación formativa busca esencialmente el papel que pueda cumplir la investigación en el aprendizaje del conocimiento.

- La investigación formativa parte del aprendizaje basado en la solución de problemas.

- Una arista del aprendizaje basado en la solución de problemas es la enseñanza por proyectos.

- Los proyectos se plantean a partir de un problema del conocimiento.

- A partir de los problemas se generan objetivos.

- Los objetivos enuncian competencias que se desprenden del método de las ciencias.

- Dichas competencias generan sistemas de tareas al interior del sistema didáctico que modelan procesos de investigación.

- La articulación de las funciones de docencia e investigación generan a través de las competencias, el desarrollo de la alta inteligencia.

\section{REFERENCIAS BIBLIOGRÁFICAS}

[1] Consejo Nacional de Acreditación. Evaluación Externa en el Contexto de la Acreditación en Colombia. Colección Documentos de reflexión No. 1.2.4.2 Acerca de la Investigación.

[ 2 ] UNESCO (1998) Conferencia Mundial sobre la Educación Superior. Declaración Mundial sobre la Educación Superior en el Siglo XXI: Visión y Acción. 9 de Octubre de 1998.

[ 3 ] Restrepo Gómez, Bernardo. "Conceptos y aplicaciones de la investigación formativa y criterios para evaluar la investigación científica en sentido estricto.

[4] Savater, Fernando (2007). La educación es base de la democracia. Los ignorantes influyen negativamente.http://www.cna.gov.co

[ 5 ] Acuerdo No. 02 de Marzo 28 de 2.008 de la Escuela Colombiana de Carreras Industriales

[6] ALVAREZ, Carlos. Didáctica. La Escuela en la Vida. La Habana, Pueblo y Educación, 1999.

[7] ALVAREZ, Carlos. La Universidad como Institución Social. La Habana, Academia, 1996.

[ 8 ] ALVAREZ, Carlos. Investigación en Educación. Inédito, 1999.

[ 9 ] ATTALI, Jacques. Milenio. Seix Barral. Santafé de Bogotá, 1992. 
[ 10 ] BACHELARD, Gaston. La Formación del Espíritu Científico. Contribución a un Psicoanálisis del Conocimiento Objetivo. Siglo Veintiuno Editores. Santafé de Bogotá, 1990.

[11] BREZINKA, Wolfgang. Los Límites de la Educación. Revista de educación. Tubingen, Instituto de Colaboración Científica,1983. \# 27.

[12] CAMPO, Rafael y RESTREPO, Mari luz. Un modelo de seminario para estudio de posgrados. Santafé de Bogotá, Pontificia Universidad Javeriana, 1993.

[13] CHEVALLARD, Yves (1991). La transposición didáctica. Del saber sabio al saber enseñado. Buenos Aires: Aique.

[ 14] DE ZUBERÍA Samper, Sergio. La cultura de la condición posmoderna. En: Conferencia: Posmodernidad y cultura en América Latina. Foro de Educación con calidad, Medellín, Seduca, 1994.

[ 15 ] DE ZUBIRÍA, Miguel y De Zubiría, Julián. Biografía del Pensamiento. Estrategias para el Desarrollo de la Inteligencia. Magistério. Santafé de Bogotá, 1996.

[16] DRUCKER, Peter. Gerencia para el Futuro. Norma. Santafé de Bogotá, 1993.

[17 ] FREIRE, Paulo. Pedagogía de la Pre-gunta. Revista Educación y Cultura. No. 11, págs. 56-60.

[18] GADAMER, Hans - George. Verdad y Método. Salamanca: Sígueme, 1997. Tomo I.

[ 19 ] GIL PÉREZ, Daniel. "El surgimiento de la didáctica de las ciencias como campo específico de conocimientos" en: Revista Educación y Pedagogía. Medellín, No. 25, Vol.11, sep-dic., 1999. p.13-65.
[ 20 ] GONZÁLEZ AGUDELO, Elvia María (2005). Sobre la hermenéutica o acerca de las múltiples lecturas de lo real. Medellín, universidad de Antioquia, s.p.i. [21] GÓMEZ BUENDİA. Hernando. ¿Para dónde va Colombia? Bogotá: tm editores, 1999

[ 22 ] JARAMILLO SALAZAR, Hernán y GÒMEZ BUENDIA, Hernando. 37 modos de hacer ciencia en América Latina. Bogotá: tm editores, 1997.

[23] NOT, Louis. Las pedagogías del conocimiento. México, fondo de la cultura económica, 1994.

[ 24 ] MISIÓN CIENCIA EDUCACIÓN Y DESARROLLO. Colombia al filo de la oportunidad. Presidencia de la República de Colombia. 1994.

[ 25] MOCKUS, Antanas y otros. Las fronteras de la escuela. Santa fe de Bogotá, Sociedad Colombiana de Pedagogía, 1994.

[ 26 ] PLAN DE DESARROLLO UNIVERSIDAD DE ANTIOQUIA 1995 2006. La Universidad para un nuevo siglo de las Luces. Consejo Superior Universidad de Antioquia, 1996.

[ 27] RODRÍGUEZ ILLERA, José Luis. Educación y comunicación. Barcelona, Paidós, 1988.

[ 28 ] UNESCO. La educación encierra un tesoro. Informe a la UNESCO de la comisión internacional sobre la educación para el siglo XXI presedida por Jaques Delors. México, Unesco, 1997. 